\title{
Explicit Solution for Cylindrical Heat Conduction
}

\author{
Kaitlyn Parsons ${ }^{* a}$, Tyler Reichanadter ${ }^{a}$, Andi Vicksman $^{a}$, Harvey Segur $^{a}$ \\ ${ }^{a}$ Department of Applied Mathematics, University of Colorado, Boulder, CO \\ Students: *kaitlyn.parsons@colorado.edu,jonathan.reichanadter@colorado.edu,andi.vicksman@colorado.edu \\ Mentor: harvey.segur@colorado.edu
}

\begin{abstract}
The heat equation is a partial differential equation that elegantly describes heat conduction or other diffusive processes. Primary methods for solving this equation require time-independent boundary conditions. In reality this assumption rarely has any validity. Therefore it is necessary to construct an analytical method by which to handle the heat equation with time-variant boundary conditions. This paper analyzes a physical system in which a solid brass cylinder experiences heat flow from the central axis to a heat sink along its outer rim. In particular, the partial differential equation is transformed such that its boundary conditions are zero which creates a forcing function in the transform PDE. This transformation constructs a Green's function, which admits the use of variation of parameters to find the explicit solution. Experimental results verify the success of this analytical method.
\end{abstract}

\section{KEYWORDS}

Heat Equation; Bessel-Fourier Decomposition; Cylindrical; Time-dependent Boundary Conditions; Orthogonality; Partial Differential Equation; Separation of Variables; Green's Functions

\section{INTRODUCTION}

Separation of variables is a versatile method for solving linear, homogenous partial differential equations (PDEs). In elementary applications, the heat equation satisfies the requirements for separation of variables. More robust physical phenomena regarding heat, however, are not always ideally isolated. In this paper we derive an explicit solution of the heat equation in a cylindrical system with non-trivial time-dependent boundary conditions in order to demonstrate an approach in which separation of variables is still applicable to an inhomogeneous form of the heat equation given an appropriate change of variables.

The heat equation is solved for a brass cylinder $(70 \% \mathrm{Cu}: 30 \% \mathrm{Zn})$ with an electrical heating unit in the center and with a chilled water bath running along the outer rim. The brass medium experiences two time-dependent boundary conditions, rendering traditional analytic PDE solving techniques inapplicable. ${ }^{1}$ A transformation is applied to the cylindrical heat equation such that the transformed boundary conditions are zero. Additionally, a forcing function is now added to the transformed heat equation as a result of the transformation. This inhomogeneous PDE can then be split into a particular and homogenous solution. The latter is solved by the method of separation of variables. The inhomogeneous is solved by variation of parameters and construction of Green's functions. ${ }^{2}$ The establishment of Fourier-Bessel decomposition via orthogonality conditions enable calculation of the coefficients in the homogeneous and particular solutions.

The brass cylinder was heated until each thermistor reached its steady-state temperature. Next the water chiller was turned on and data were collected at each thermistor as the temperature changed with respect to time. Data from the outer and inner thermistors were input as the boundary conditions in the theoretical model. Similarly, the steady-state temperatures measured on all six thermistors were input as the initial condition in the theoretical model. The remaining set of data for the four inner thermistors served as a comparison for the explicit solution derived in our paper. 


\section{Experimental Apparatus}

The experimental setup consisted of a brass cylinder of radius $150 \mathrm{~mm}$ and height $35 \mathrm{~mm}$ manufactured by Armfield Ltd. (Ringwood Hampshire, U.K.). An electric heating unit located in the center of the cylinder transferred heat into the apparatus while cool water from the chilling bath flowed simultaneously around the outer edge. Seven temperature probes spaced evenly apart in a radial path collected data deep within the brass cylinder over time. The probes were located at $10 \mathrm{~mm}$ increments starting with temperature-thermistor 1 at $10 \mathrm{~mm}$ and ending with the last temperature-thermistor at $70 \mathrm{~mm}$. The thermistor located at $30 \mathrm{~mm}$ failed to collect data so it was not used in the experiment or data analysis. For clarity, the thermistors were renumbered 1 through 6, skipping the failed thermistor. Thus, thermistor 6 refers to the thermistor located at $70 \mathrm{~mm}$. The innermost probe, labeled temperature-thermistor 1 , recorded the boundary condition at $R_{i}$, corresponding to the inner boundary condition $v\left(R_{i}, t\right)=g(t)$. The outermost probe, labeled temperature-thermistor 6, recorded the boundary condition at $R_{o}$, corresponding respectively to the outer boundary condition $v\left(R_{o}, t\right)=h(t)$.

Figure 1 portrays the apparatus with the skipped thermistor crossed out:

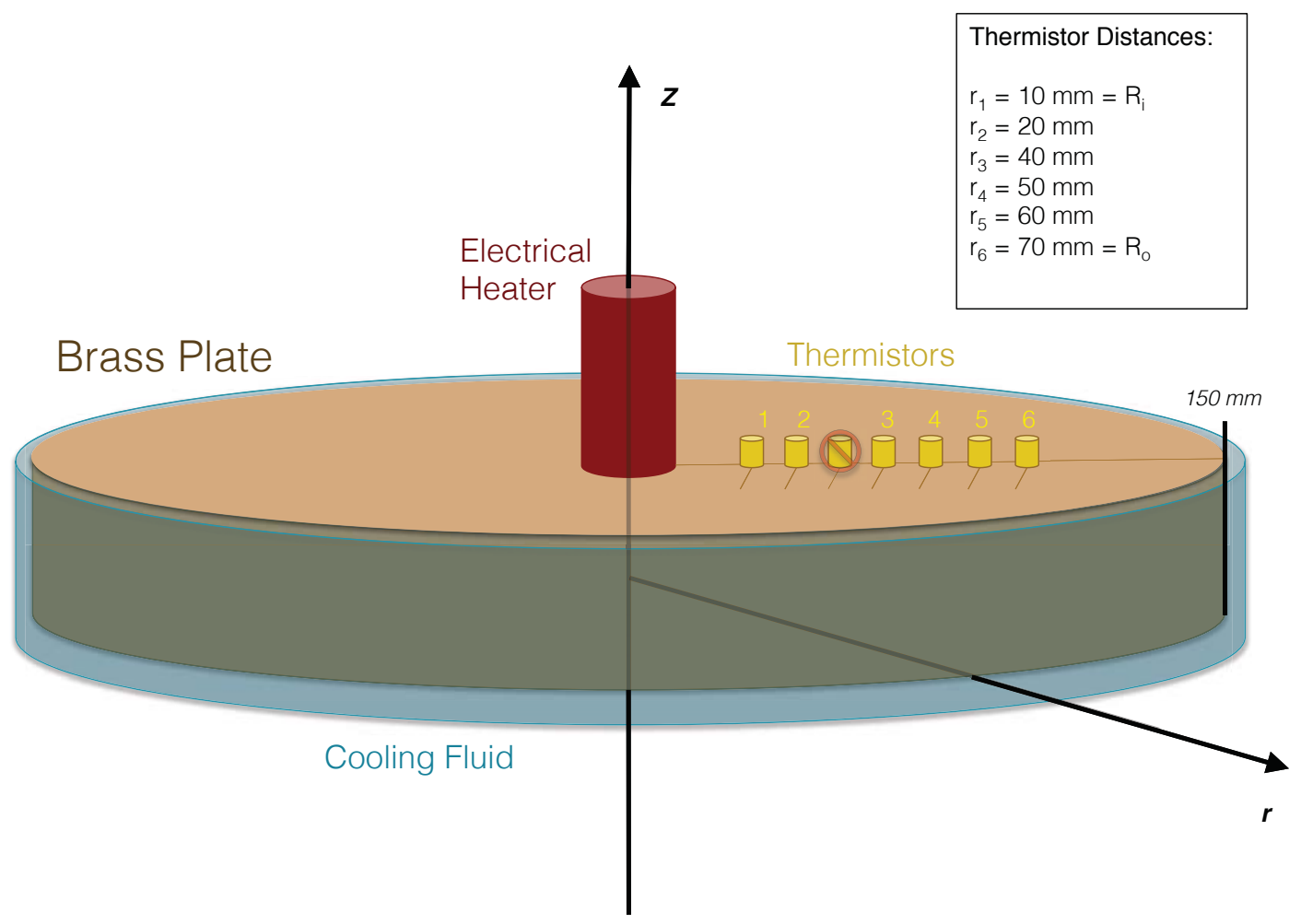

Figure 1. Physical setup for thermal experiment and data collection.

Before collecting data, the water was chilled to $10^{\circ} \mathrm{C}$. In addition, the power regulator was set to approximately 15 Watts and was allowed to reach a steady state. Once this occurred the pump connected from the water chiller to the apparatus was turned on allowing water to flow around the outside of the cylinder. After turning this on, thermistor data were collected every six seconds. About four minutes later, the experiment reached steady state and the data collection was stopped. Two trials were conducted with consistent results. 


\section{Mathematical Model}

The standard heat equation is

$$
\frac{\partial v}{\partial t}-\kappa \nabla^{2} v=0
$$

Equation 1.

where $v(\mathbf{r}, t)$ is the temperature at point $\mathbf{r}$ and time $t, \kappa$ is the thermal diffusivity, and $\nabla^{2} v(\mathbf{r}, t)$ is the Laplacian of the function $v$ or the divergence of the gradient of $v$.

The temperature varies with radial distance from the boundaries while remaining uniform in the $z$ direction so the most appropriate coordinate system for this analysis is polar coordinates. Additionally, the apparatus is radially symmetric, thus the temperature is only a function of radius $r$. This results in the following equation

$$
\begin{gathered}
\frac{\partial v}{\partial t}=\kappa\left[\frac{1}{r} \frac{\partial}{\partial r}\left(r \frac{\partial v}{\partial r}\right)\right]+F(r, t), \quad R_{i}<r<R_{o}, \quad t>0 \\
v\left(R_{i}, t\right)=g(t) \\
v\left(R_{o}, t\right)=h(t) \\
v(r, 0)=V(r) \quad R_{i}<r<R_{o},
\end{gathered}
$$

Equation 2.

where $R_{i}$ is the radius of the inner probe, $R_{o}$ is the radius of the outer probe, and $F(r, t)$ is the forcing function induced by the central heater and the outer rim heat sink. For brass the thermal diffusivity is $\kappa=37.52 \times 10^{-6} \frac{\mathrm{m}^{2}}{\mathrm{~s}}$. ${ }^{3}$ The functions $g(t)$ and $h(t)$ represent the boundary conditions at the inner probe and outer probe respectively and $V(r)$ is the initial condition.

\section{ANALYTICAL SOLUTION}

Currently the non-zero, time-dependent boundary conditions prevent the use of Fourier's decomposition method. ${ }^{4}$ The solutions to (Equation 2) do not form a closed function space; a linear combination of eigenfunctions satisfying the differential equation in (Equation 2) will not necessarily satisfy the conditions associated with (Equation 2).

Thus the first step is to change variables so that the boundary conditions are identically zero. The characteristic properties of Green's function are then utilized to find the explicit solution. Green's function enables linear boundary-value problems to be solved by transforming the original problem so that the solution depends exclusively on the boundary conditions and the differential equation. ${ }^{2}$ There are five characteristic properties of Green's function. ${ }^{2}$ In order to use Green's function we must transform our problem so that the boundary conditions are identically zero. This is accomplished by defining $z(r, t)$ as $z(r, t)=v(r, t)-\tau_{1}(r) g(t)-\tau_{2}(r) h(t)$.

In order for $z(r, t)$ to exist in a closed function space it is required that $z\left(R_{i}, t\right)=z\left(R_{o}, t\right)=0$ so the following must be true: $\tau_{1}\left(R_{i}\right)=1, \tau_{1}\left(R_{o}\right)=0, \tau_{2}\left(R_{i}\right)=0, \tau_{2}\left(R_{o}\right)=1$. These functions are defined as:

$$
\begin{aligned}
\tau_{1}(r) & =\frac{\ln (r)-\ln \left(R_{o}\right)}{\ln \left(R_{i}\right)-\ln \left(R_{o}\right)} \\
\tau_{2}(r) & =\frac{\ln \left(R_{i}\right)-\ln (r)}{\ln \left(R_{i}\right)-\ln \left(R_{o} 1\right)}
\end{aligned}
$$

Substituting this back into (Equation 2) results in a new differential equation that can be solved using separation of variables, since the modified boundary conditions of (Equation 2) form a closed function space.

These particular choices for $\tau_{1}$ and $\tau_{2}$ are convenient since their derivatives with respect to $r$ become $\pm \frac{1}{r}$. This is useful because on the right hand side of the PDE the solution $z(r, t)$ is differentiated, multiplied by $r$, and then differentiated 
again with respect to $r$. So the derivative of $\tau_{1}$ and $\tau_{2}$ of $\pm \frac{1}{r}$ gets multiplied by $r$, making the derivatives constant which results with the second derivatives equal to zero. Therefore, the forcing term only contains time-derivative information. The problem now becomes:

$$
\begin{gathered}
\frac{\partial z}{\partial t}=\kappa\left[\frac{1}{r} \frac{\partial}{\partial r}\left(r \frac{\partial z}{\partial r}\right)\right]+\hat{F}(r, t), \quad R_{i}<r<R_{o}, \quad t>0 \\
z\left(R_{i}, t\right)=0 \\
z\left(R_{o}, t\right)=0 \\
z(r, 0)=\hat{V}(r) \quad R_{i}<r<R_{o},
\end{gathered}
$$

where

$$
\begin{aligned}
& \hat{F}(r, t)=F(r, t)-\left[\frac{\ln (r)-\ln \left(R_{o}\right)}{\ln \left(R_{i}\right)-\ln \left(R_{o}\right)}\right] \frac{d g}{d t}-\left[\frac{\ln \left(R_{i}\right)-\ln (r)}{\ln \left(R_{i}\right)-\ln \left(R_{o}\right)}\right] \frac{d h}{d t} \\
& \hat{V}(r)=V(r)-\left[\frac{\ln (r)-\ln \left(R_{o}\right)}{\ln \left(R_{i}\right)-\ln \left(R_{o}\right)}\right] g(0)-\left[\frac{\ln \left(R_{i}\right)-\ln (r)}{\ln \left(R_{i}\right)-\ln \left(R_{o}\right)}\right] h(0)
\end{aligned}
$$

The next logical step is to solve for $z(r, t)$ of the form $z(r, t)=z_{h}(r, t)+z_{p}(r, t)$ where $z_{h}(r, t)$ is the homogenous solution and $z_{p}(r, t)$ is the particular solution. ${ }^{5}$ By applying the method of variation of parameters, the resulting form of the particular solution will be similar to the homogenous solution. In order to find the particular solution, the homogenous solution must therefore be solved for first.

\section{Homogenous Solution}

The PDE corresponding to the homogenous solution is by construction:

$$
\frac{\partial z_{h}}{\partial t}=\kappa\left[\frac{1}{r} \frac{\partial z_{h}}{\partial r}+\frac{\partial^{2} z_{h}}{\partial r^{2}}\right]
$$

Equation 4.

With a homogenous PDE and $z_{h}\left(R_{i}, t\right)=z_{h}\left(R_{o}, t\right)=0$ boundary conditions, $z_{h}(r, t)$ is solved by separations of variables. The solution is redefined by $z_{h}(r, t)=U(r) S(t)$. Following the process of separation of variables leads to:

$$
\frac{1}{\kappa} \frac{S^{\prime}(t)}{S(t)}=\frac{1}{r} \frac{U^{\prime}(r)}{U(r)}+\frac{U^{\prime \prime}(r)}{U(r)}=-\lambda
$$

in which $\lambda$ is an eigenvalue of the equation. ${ }^{6}$

\section{Case 1: $\lambda<0$}

For the case in which the eigenvalue is negative $\lambda \equiv-q^{2}$ the differential equation becomes

$$
U^{\prime \prime}(r)+\frac{1}{r} U^{\prime}(r)-q^{2} U(r)=0
$$

Equation 5.

This second-order differential equation is a specific form of Bessel's Equation which is shown below: ${ }^{7}$

$$
y^{\prime \prime}(x)+(d-1) \frac{1}{x} y^{\prime}(x)-\left(\nu-\frac{\mu}{x^{2}}\right) y(x)=0
$$


Notice the choice of $d=2, \mu=0$, and $\nu=q^{2}$ recovers the radial equation. With these constraints the solution to the radial equation is of the form $F(x)=C_{1} K_{\mu}(x \sqrt{\nu})+C_{2} I_{\mu}(x \sqrt{\nu})$ where $K_{\mu}$ and $I_{\mu}$ are the modified Bessel functions of the first and second kind which simplify in this application to: ${ }^{8}$

$$
U(r)=C_{1} K_{0}(r q)+C_{2} I_{0}(r q)
$$

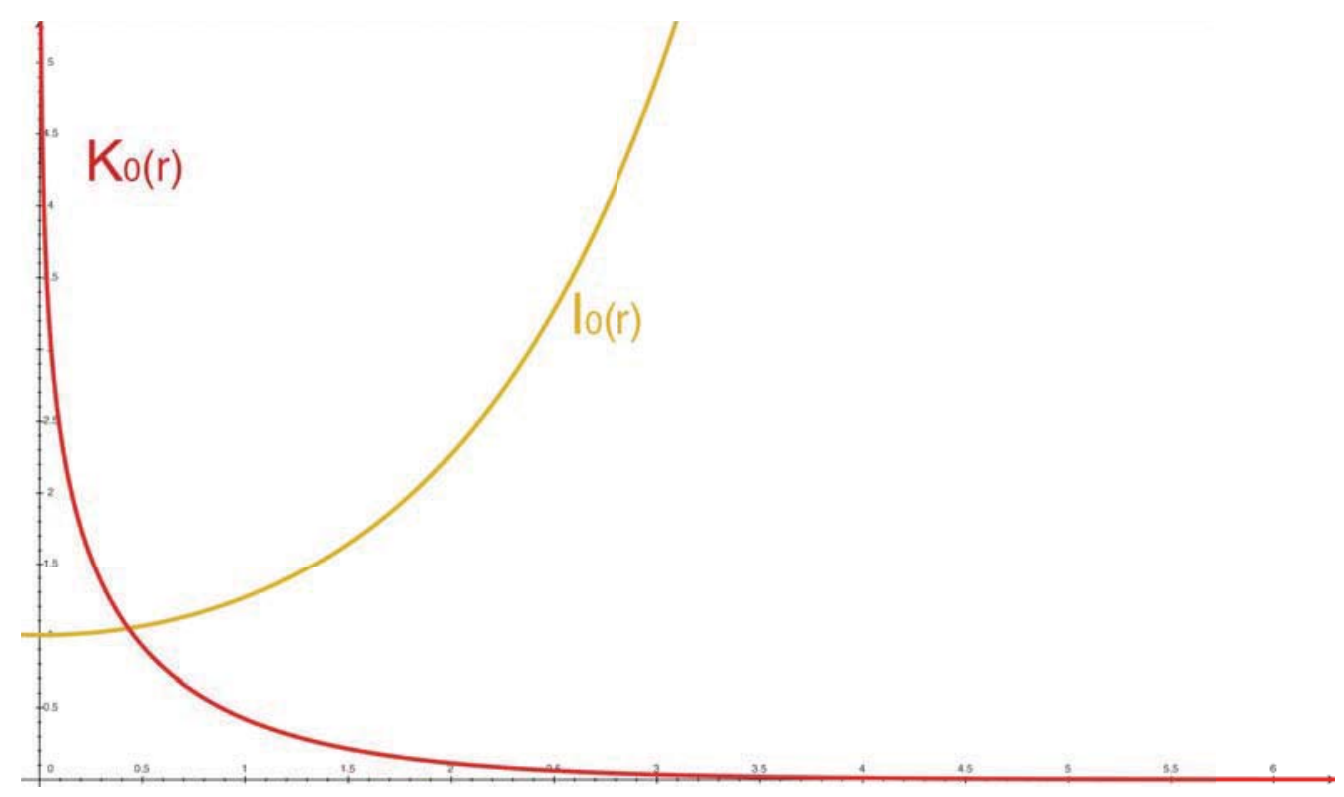

Figure 2. Modified Bessel functions of the first and second kind $I_{0}, K_{0}\left(d=2, \mu=0\right.$, and $\left.\nu=q^{2}\right)$.

Substituting in boundary conditions results in

$$
\begin{gathered}
U\left(R_{i}\right)=C_{1} K_{0}\left(R_{i} q\right)+C_{2} I_{0}\left(R_{i} q\right)=0 \\
U\left(R_{o}\right)=C_{1} K_{0}\left(R_{o} q\right)+C_{2} I_{0}\left(R_{o} q\right)=0
\end{gathered}
$$

The two equations above form a matrix with a solution of the zero vector. Since $K_{0}$ and $I_{0}$ are linearly independent functions it follows that the null space is empty and $C_{1}=C_{2}=0$, rendering the trivial solution.

Case 2: $\lambda=0$

For this case $\lambda \equiv 0$. Substituting this into the differential equation and simplifying results in:

$$
\frac{U^{\prime \prime}(r)}{U^{\prime}(r)}=-\frac{1}{r}
$$

Equation 6.

The first step in solving (Equation 6) is to integrate both sides, producing the expression $\ln \left(U^{\prime}(r)\right)=-\ln (r)+C$. From here $U^{\prime}(r)=e^{\ln (1 / r)+C}=C_{1} \frac{1}{r}$. After integrating once more the solution is found to be

$$
U(r)=C_{1} \ln r+C_{2} .
$$

Substituting in boundary conditions results in

$$
\begin{aligned}
& U\left(R_{i}\right)=C_{1} \ln R_{i}+C_{2}=0 \\
& U\left(R_{o}\right)=C_{1} \ln R_{o}+C_{2}=0
\end{aligned}
$$

From this, $C_{2}=-C_{1} \ln R_{i}$ and $C_{2}=-C_{1} \ln R_{o}$. Combined these become $0=C_{1}\left(\ln R_{o}-\ln R_{i}\right)$ which implies $C_{1}=$ $C_{2}=0$ resulting in the trivial solution. 


\section{Case 3: $\lambda>0$}

Finally for $\lambda \equiv m^{2}$ the resulting differential equation is

$$
U^{\prime \prime}(r)+\frac{1}{r} U^{\prime}(r)+m^{2} U(r)=0
$$

Equation 7.

This third case produces a radial ODE almost identical to the equation for the negative eigenvalue. The key difference between the two is that $q^{2}=-m^{2}$ so the linearly independent solutions to this equation are the Bessel functions of the first and second kind: ${ }^{9}$

$$
U(r)=C_{1} J_{0}(r m)+C_{2} Y_{0}(r m)
$$

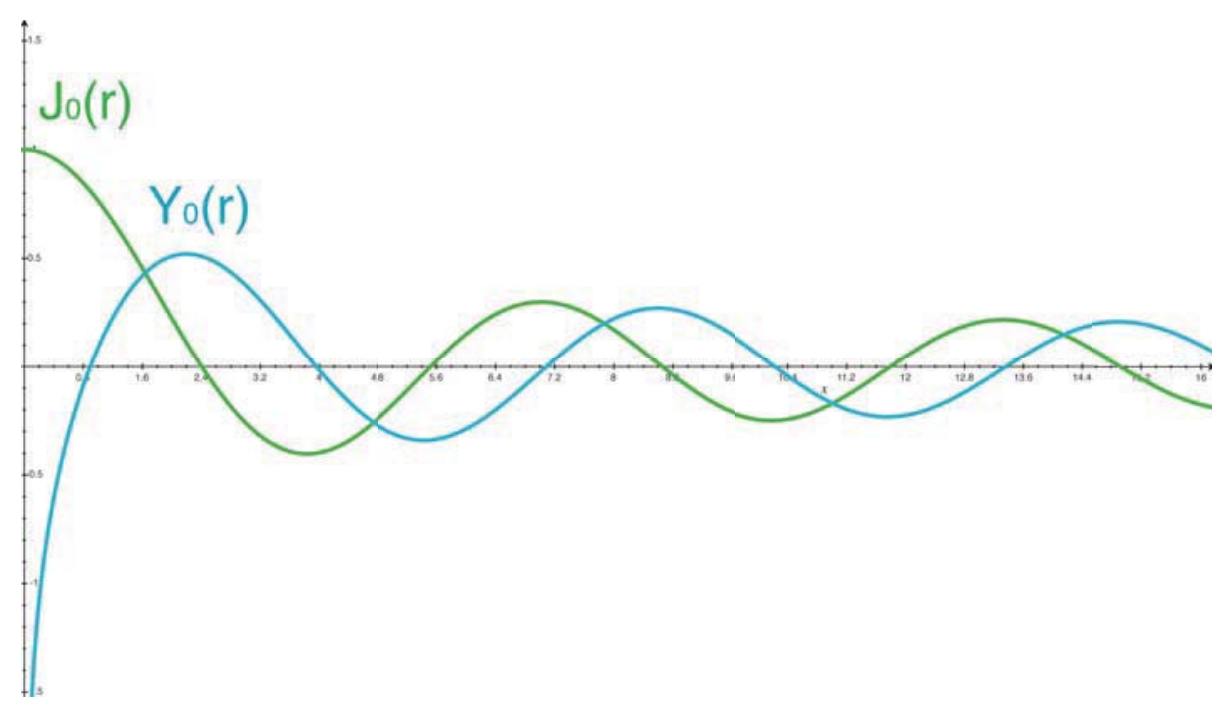

Figure 3. Modified Bessel functions of the first and second kind $J_{0}, Y_{0}\left(d=2, \mu=0\right.$, and $\left.\nu=-m^{2}\right)$.

Substituting in boundary conditions results in

$$
\begin{gathered}
U\left(R_{i}\right)=C_{1} J_{0}\left(R_{i} m\right)+C_{2} Y_{0}\left(R_{i} m\right)=0 \\
U\left(R_{o}\right)=C_{1} J_{0}\left(R_{o} m\right)+C_{2} Y_{0}\left(R_{o} m\right)=0
\end{gathered}
$$

\section{Equation 8.}

Equation 9.

The boundary condition equations (Equation 8) and (Equation 9) establish that $\frac{C_{2}}{C_{1}}=-\frac{J_{0}\left(R_{i} m\right)}{Y_{0}\left(R_{i} m\right)}=-\frac{J_{0}\left(R_{o} m\right)}{Y_{0}\left(R_{o} m\right)}$. Given the oscillatory behavior of the Bessel equations of the first and second kind, there exists an infinite set of nontrivial coefficients. We will denote this set by $\left\{m_{n}\right\}_{n=1}^{\infty}$ and solve for terms in this set using the equality of $\frac{C_{2}}{C_{1}}$. Similar to the equations in case two, equations (Equation 8) and (Equation 9) form a matrix. The correct eigenvalues can be determined as they make the matrix singular and its determinant zero. Given the experimental values of $R_{i}$ and $R_{o}$, the first five terms are provided in table Table 1:

The $m_{n}$ values are the square roots of the eigenvalues satisfying the boundary conditions.

\section{Solution}

As a result from solving the radial equation, the set of eigenvalues is strictly positive and defined by $\lambda_{n}=m_{n}^{2}$ where $n=1,2,3 \ldots$ as portrayed in table Table 1 . Now the temporal equation $S^{\prime}(t)=\kappa m_{n}^{2} S(t)$ is easily derived resulting in 


\begin{tabular}{|c|c|}
\hline $\mathbf{n}$ & $\mathbf{m}_{\mathbf{n}}$ \\
\hline 1 & 50.3 \\
\hline 2 & 103.4 \\
\hline 3 & 156.1 \\
\hline 4 & 208.7 \\
\hline 5 & 261.2 \\
\hline
\end{tabular}

Table 1. Table of first five eigenvalues.

$S_{n}(t)=C_{n} e^{-m^{2} \kappa t}$. Putting this together with $U(r)$ produces the homogeneous solution:

$$
z_{h}(r, t)=\sum_{n=1}^{\infty} A_{n}\left[J_{0}\left(m_{n} r\right)+B_{n} Y_{0}\left(m_{n} r\right)\right] e^{-m_{n}^{2} \kappa t}
$$

Equation 10.

where the new coefficient $B_{n} \equiv \frac{C_{2}}{C_{1}}$ is calculated from $-\frac{C_{2}}{C_{1}}=\frac{J_{0}\left(R_{i} m_{n}\right)}{Y_{0}\left(R_{i} m_{n}\right)}=\frac{J_{0}\left(R_{o} m_{n}\right)}{Y_{0}\left(R_{o} m_{n}\right)}$ and $A_{n}$ is a free constant.

Particular Solution of the PDE

In order to solve for the particular solution $z_{p}(r, t)$ of the transformed PDE, we use the technique of variation of parameters. ${ }^{10}$ The free constants $A_{n}$ in the homogeneous solution (Equation 10) are extended to have time-dependency $A_{n}(t)$ :

$$
z_{p}(r, t)=\sum_{n=1}^{\infty} U_{n}(r) S_{n}(t)=\sum_{n=1}^{\infty} A_{n}(t)\left[J_{0}\left(m_{n} r\right)+B_{n} Y_{0}\left(m_{n} r\right)\right] e^{-m_{n}^{2} \kappa t}
$$

Equation 11.

Observe that equation (Equation 11)is a generalization of equation (Equation 10). The transformed forcing function is re-introduced into the differential equation shown below:

$$
\frac{\partial z_{p}}{\partial t}-\kappa\left[\frac{1}{r} \frac{\partial z_{p}}{\partial r}+\frac{\partial^{2} z_{p}}{\partial r^{2}}\right]=\hat{F}(r, t)
$$

Equation 12.

When $z_{p}(r, t)$ of the form given by (Equation 11) is substituted into the differential equation (Equation 12), the product rule of derivatives applies and results in two terms from the partial derivative with respect to $t$. The time-derivative term in (Equation 12) is similar to the homogenous solution, only varying by the time-dependent coefficients $A_{n}(t)$. Since this term mimics the form of the homogeneous solution it therefore similarly satisfies the homogeneous PDE and cancels to zero as given by: $\frac{\partial z_{h}}{\partial t}-\kappa\left[\frac{1}{r} \frac{\partial z_{h}}{\partial r}+\frac{\partial^{2} z_{h}}{\partial r^{2}}\right]=0$. The radial derivative terms of (Equation 12) are a consequence of the product rule applied to $\frac{\partial}{\partial t}\left[z_{p}(r, t)\right]=U(r) \frac{d}{d t}\left[A_{n}(t) e^{-m_{n}^{2} \kappa t}\right]$, so the reduced form of (Equation 12) is given by:

$$
\hat{F}(r, t)=\sum_{n=1}^{\infty} A_{n}^{\prime}(t)\left[J_{0}\left(m_{n} r\right)+B_{n} Y_{0}\left(m_{n} r\right)\right] e^{-m_{n}^{2} \kappa t}
$$

Equation 13.

(Equation 13) for the transformed forcing function provides an avenue by which to calculate the particular solution coefficients $A_{n}(t) . \hat{F}(r, t)$ is calculated from the experimental boundary conditions, so we may determine each $A_{n}^{\prime}(t)$ by expressing it in the basis of adjusted Bessel functions $\left[J_{0}\left(m_{n} r\right)+B_{n} Y_{0}\left(m_{n} r\right)\right]$. This decomposition into the basis functions requires identifying the orthogonality conditions of these functions which will be developed in the next section. After finding $A_{n}^{\prime}(t)$, the $A_{n}(t)$ terms are determined by integrating with respect to time from 0 to $t: A_{n}(t)=\int_{0}^{t} A_{n}^{\prime}(\tau) d \tau$. 
The full transformed solution then becomes:

$$
z(r, t)=\sum_{n=1}^{\infty}\left[A_{n}(t)+A_{n}\right]\left[J_{0}\left(m_{n} r\right)+B_{n} Y_{0}\left(m_{n} r\right)\right] e^{-m_{n}^{2} \kappa t}
$$

Equation 14.

First we solve for the homogeneous constants. If $z(r, t)$ is evaluated at $t=0$ the solution becomes:

$$
z(r, 0)=\sum_{n=1}^{\infty}\left[A_{n}(0)+A_{n}\right]\left[J_{0}\left(m_{n} r\right)+B_{n} Y_{0}\left(m_{n} r\right)\right] e^{-m_{n}^{2} \kappa(0)} \equiv \hat{V}(r)
$$

This expression quickly simplifies, starting with the fact that $e^{-m_{n}^{2} \kappa(0)}=1$. Additionally, as a result of the particular solution's construction, $A_{n}(0)=0$ for $n=1,2,3 \ldots$ since the bounds of integration are $(0,0)$. The remaining expression elicits a technique for retrieving the $A_{n}$ terms

$$
\hat{V}(r)=\sum_{n=1}^{\infty} A_{n}\left[J_{0}\left(m_{n} r\right)+B_{n} Y_{0}\left(m_{n} r\right)\right]
$$

Equation 15.

using a similar basis decomposition method that is discussed in the following section, in which we will show how to explicitly calculate $A_{n}^{\prime}(t)$ and $A_{n}$ from (Equation 13) and (Equation 15) respectively.

\section{Bessel-Fourier Decomposition}

To find the $A_{n}^{\prime}(t)$ and $A_{n}$ terms it is essential to establish the conditions of orthogonality for the basis functions $Q_{n}(r)=$ $J_{0}\left(m_{n} r\right)+B_{n} Y_{0}\left(m_{n} r\right) .{ }^{10}$ The derivation requires analysis based on the general Sturm-Liouville equation. Such conditions emerge from the substitution of the basis functions into the radial ODE (Equation 7):

$$
\frac{d}{d r}\left[r \frac{d Q_{n}}{d r}\right]+r m^{2} Q_{n}=0 .
$$

Equation 16.

Note that the $r$ dependence in $Q_{n}$ still exists, yet $Q_{n}(r)$ is notationally simplified to $Q_{n}$ in this derivation for convenience. Two equations $Q_{p}$ and $Q_{s}$ are established with eigenvalues $\lambda_{p}=m_{p}^{2}$ and $\lambda_{s}=m_{s}^{2}$. This generates the two following ODEs:

$$
\begin{aligned}
& \frac{d}{d r}\left[r \frac{d Q_{s}}{d r}\right]+r m_{s}^{2} Q_{s}=0 \\
& \frac{d}{d r}\left[r \frac{d Q_{p}}{d r}\right]+r m_{p}^{2} Q_{p}=0
\end{aligned}
$$

Multiplying the top equation by $Q_{p}$ and the second by $Q_{s}$ results in:

$$
\begin{aligned}
& Q_{p} \frac{d}{d r}\left[r \frac{d Q_{s}}{d r}\right]+r m_{s}^{2} Q_{s} Q_{p}=0 \\
& Q_{s} \frac{d}{d r}\left[r \frac{d Q_{p}}{d r}\right]+r m_{p}^{2} Q_{p} Q_{s}=0
\end{aligned}
$$

Equation 17.

Equation 18.

Then subtracting (Equation 18) from (Equation 17) and expanding results in

$$
r Q_{p} Q_{s}^{\prime \prime}-r Q_{s} Q_{p}^{\prime \prime}+Q_{p} Q_{s}^{\prime}-Q_{s} Q_{p}^{\prime}+r m_{s} Q_{s} Q_{p}-r m_{p} Q_{p} Q_{s}=0
$$

The next step is to integrate this equation.

$$
\int_{R_{i}}^{R_{o}} r Q_{p} Q_{s}^{\prime \prime} d r-\int_{R_{i}}^{R_{o}} r Q_{s} Q_{p}^{\prime \prime} d r+\int_{R_{i}}^{R_{o}}\left(Q_{p} Q_{s}^{\prime}-Q_{s} Q_{p}^{\prime}\right) d r+\left(m_{s}-m_{p}\right) \int_{R_{i}}^{R_{o}} r Q_{p} Q_{s} d r=0
$$


With the application of the integration by parts theorem on the first two integrals and the boundary conditions $z\left(R_{i}, t\right)=0$ and $z\left(R_{o}, t\right)=0$, the entire expression collapses to:

$$
\left(m_{s}-m_{p}\right) \int_{R_{i}}^{R_{o}} r Q_{p} Q_{s} d r=0 .
$$

Equation 19.

Only when $m_{s}-m_{p}=0$ is the integral not necessarily zero. Therefore it is concluded that

$$
\int_{R_{i}}^{R_{o}} r Q_{p} Q_{s} d r=\left\{\begin{array}{rll}
0 & \text { if } & p \neq s \\
L_{p} & \text { if } & p=s
\end{array}\right.
$$

Equation 20.

where $L_{p}$ is a numerically calculated constant. (Equation 20) defines the orthogonality condition required for calculating the terms in the Fourier-Bessel decomposition of a function.

Both the transformed forcing function $\hat{F}(r, t)$ and the transformed initial condition $\hat{V}(r)$ are expressible as a linear combination of the basis functions:

$$
\begin{gathered}
\hat{V}(r)=\sum_{k=1}^{\infty} A_{n} Q_{n}\left(m_{n} r\right) \\
\hat{F}(r, t)=\sum_{k=1}^{\infty} A_{n}^{\prime}(t) Q_{n}\left(m_{n} r\right) e^{m_{n}^{2} \kappa t}
\end{gathered}
$$

The orthogonality condition identified in (Equation 20) provides a method of solving for the coefficients. The transformed initial condition and forcing function are linear combinations of the basis functions, so it follows that

$$
\begin{gathered}
A_{n}=\frac{1}{L_{n}} \int_{R_{i}}^{R_{o}} r Q_{n}\left(m_{n} r\right) \hat{V}(r) d r \\
A_{n}^{\prime}(t)=\frac{1}{L_{n}} e^{m_{n}^{2} \kappa t} \int_{R_{i}}^{R_{o}} r Q_{n}\left(m_{n} r\right) \hat{F}(r, t) d r
\end{gathered}
$$

Equation 21.

Equation 22.

From (Equation 22), we find $A_{n}(t)=\int_{0}^{t} A_{n}^{\prime}(\tau) d \tau$.

Solution Summary

Putting all of the above sections together leads to the general solution of the transformed PDE (Equation 3),

$$
z(r, t)=\sum_{n=1}^{\infty}\left[A_{n}(t)+A_{n}\right]\left[J_{0}\left(m_{n} r\right)+B_{n} Y_{0}\left(m_{n} r\right)\right] e^{-m_{n}^{2} \kappa t}
$$

with the following definitions: 


$$
\begin{aligned}
& A_{n}=\frac{1}{L_{n}} \int_{R_{i}}^{R_{o}} r Q_{n}\left(m_{n} r\right) \hat{V}(r) d r \\
& A_{n}(t)=\frac{1}{L_{n}} \int_{0}^{t} e^{m_{n}^{2} \kappa t} \int_{R_{i}}^{R_{o}} r Q_{n}\left(m_{n} r\right) \hat{F}(r, \tau) d r d \tau \\
& L_{n}=\int_{R_{i}}^{R_{o}} r Q_{n}\left(m_{n} r\right)^{2} d r \\
& Q_{n}\left(m_{n} r\right)=J_{0}\left(m_{n} r\right)+B_{n} Y_{0}\left(m_{n} r\right) \\
& B_{n}=-\frac{J_{0}\left(m_{n} R_{i}\right)}{Y_{0}\left(m_{n} R_{i}\right)} \\
& m_{n}:=\text { solutions to } \frac{J_{0}\left(m_{n} R_{o}\right)}{J_{0}\left(m_{n} R_{i}\right)}-\frac{Y_{0}\left(m_{n} R_{o}\right)}{Y_{0}\left(m_{n} R_{i}\right)}=0 \text { from the BCs of (Equation 7) ODE }
\end{aligned}
$$

The known parameters for this problem are the inner and outer radii $R_{i} \& R_{o}$, the Bessel functions $J_{0}(x) \& Y_{0}(x)$, the time-varying boundary equations $g(t) \& h(t)$, and the initial condition $V(r)$. With this collective information regarding the experiment, the temperature of the cylinder is numerically calculated by the elegant formula below, which is a consequence of the transformation $z(r, t)=v(r, t)-\tau_{1}(r) g(t)-\tau_{2}(r) h(t)$ originally stated:

$$
v(r, t)=\sum_{n=1}^{\infty}\left[\left[A_{n}(t)+A_{n}\right] Q_{n}\left(m_{n} r\right) e^{-m_{n}^{2} \kappa t}\right]+\frac{\ln (r)-\ln \left(R_{o}\right)}{\ln \left(R_{i}\right)-\ln \left(R_{o}\right)} g(t)+\frac{\ln \left(R_{i}\right)-\ln (r)}{\ln \left(R_{i}\right)-\ln \left(R_{o}\right)} h(t)
$$




\section{COMPARISON OF EXPERIMENTAL AND THEORETICAL RESULTS}

So far this report has developed the tools necessary to construct an explicit solution to the cylindrical heat equation with time-variant boundary conditions. In order to use these tools, the derived formulas require information of the physical experiment. This information includes the diffusivity constant of the brass cylinder $\left(\kappa=37.52 \times 10^{-6} \frac{\mathrm{m}^{2}}{\mathrm{~s}}\right)$, the initial condition function $V(r)$ between $R_{i}$ and $R_{o}$, the inner boundary condition function $g(t)$, and the outer boundary condition function $h(t)$. To acquire the functions $V(r), g(t)$, and $h(t)$, we collected two trials of temperature readings at each thermistor after the coolant was introduced. From these data we could determine $V(r)$ by fitting a polynomial to the initial temperature readings at each thermistor, $g(t)$ by fitting a polynomial to the innermost thermistor readings during the duration of the experiment, and $h(t)$ by fitting a polynomial to the outermost thermistor readings. The remaining temperature data are not necessary for the construction of the theoretical model, but may still serve as a means to compare the developed mathematical model with experiment. Since two trials were conducted we will build two mathematical models and compare each model with their respective data sets.

The experimental data collection began once the heater was turned on. Once the brass cylinder reached a steady temperature state the coolant was introduced to the system. Our model is developed based on the the cooling process and therefore the initial and boundary conditions are fit to the data corresponding to when the coolant was engaged. For trial one this occurred at $t=0.3$ minutes and for trial two this occurred at $t=1.0$ minutes.

\section{Solving for Initial and Boundary Conditions}

Two of the necessary inputs for the theoretical model are the two time-varying boundary conditions. The inner-radius boundary condition $g(t)$ was recorded by thermistor 1 located at the innermost radius $R_{i}=10 \mathrm{~mm}$ while the coolant was engaged. Similarly $h(t)$ is recorded by the outer thermistor-temperature probe 6 located at $R_{o}=70 \mathrm{~mm}$ from the axis center. We obtained the boundary condition functions $g(t)$ and $h(t)$ by fitting a sixth-order polynomial expression to the data (Figure 4 and 5). A sixth order fit was chosen as it was the lowest order polynomial to accurately fit the initial boundary behavior.

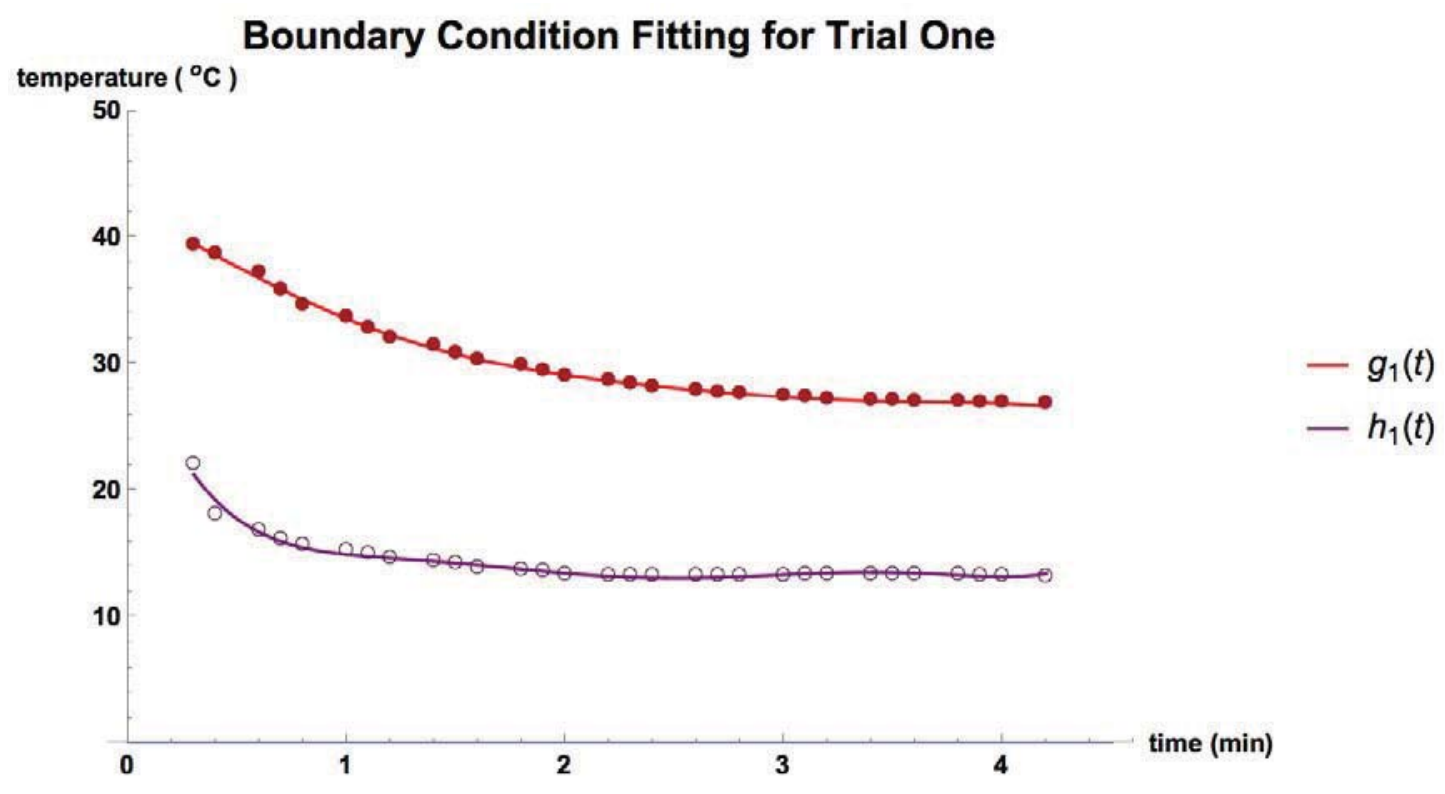

Figure 4. Trial 1 boundary condition fitting. The inner-radius boundary condition $g(t)$ was recorded by thermistor 1 located at the innermost radius $R_{i}=10 \mathrm{~mm}$. The outer-most boundary condition $h(t)$ was recorded by thermistor 6 located at $R_{o}=70 \mathrm{~mm}$ from the axis center. 


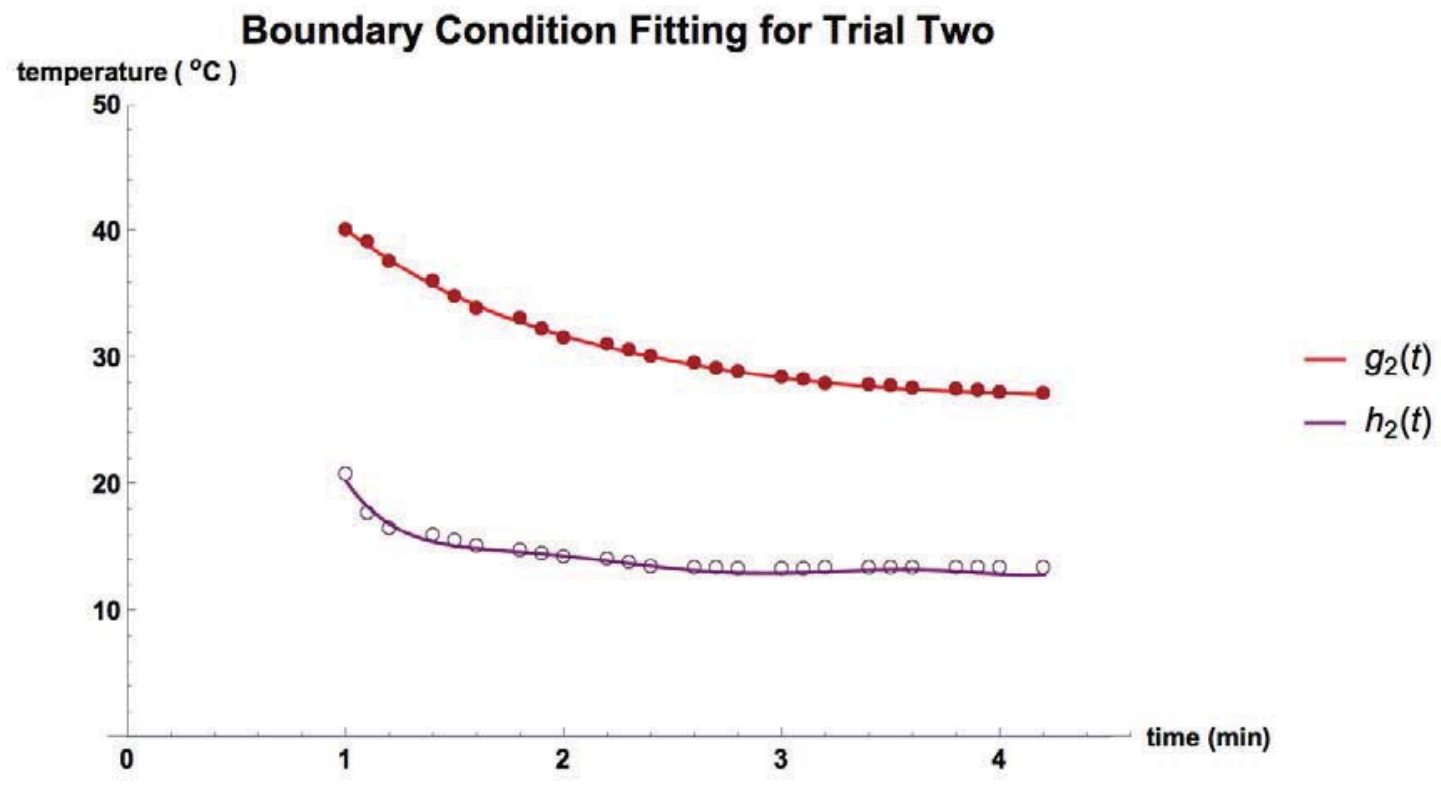

Figure 5. Trial 2 boundary condition fitting. The inner-radius boundary condition $g(t)$ was recorded by thermistor 1 located at the innermost radius $R_{i}=10 \mathrm{~mm}$. The outer-most boundary condition $h(t)$ was recorded by thermistor 6 located at $R_{o}=70 \mathrm{~mm}$ from the axis center.

Attaining the initial condition requires a similar approach as used for the boundary conditions. The initial condition function $V(r)$ for both trials was obtained by fitting a quadratic polynomial to the data, which was the lowest order polynomial to accurately represent the initial behavior. As opposed to fitting a function to the data from one thermistor, the initial condition $V(r)$ is attained by fitting the quadratic to the initial probe measurements across all thermistors as a function of radial distance from the center (Figure 6 and 7). These initial condition functions are fit to the data at $t=0.3$ minutes and $t=1.0$ minutes for trials one and two respectively when the coolant was added.

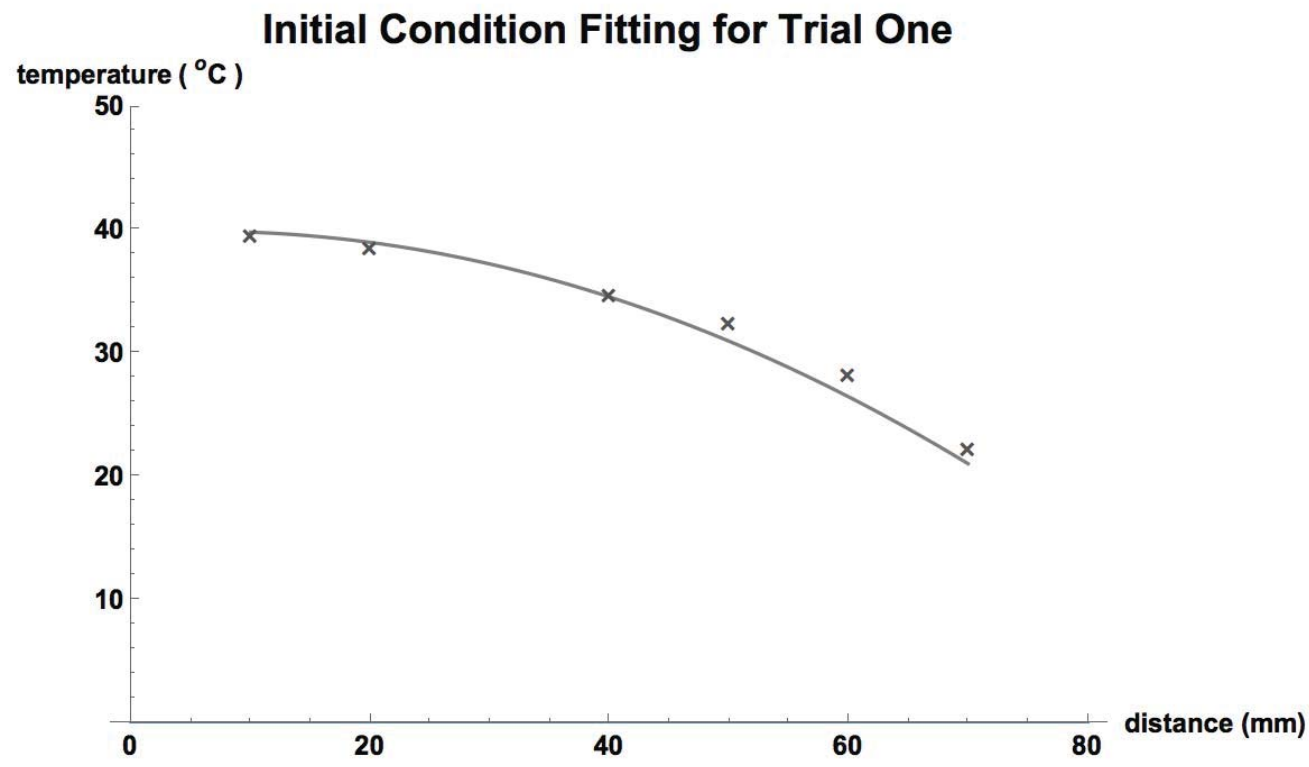

Figure 6. Trial 1 initial condition fitting from initial probe measurements across all thermistors as a function of radial distance from the center. 


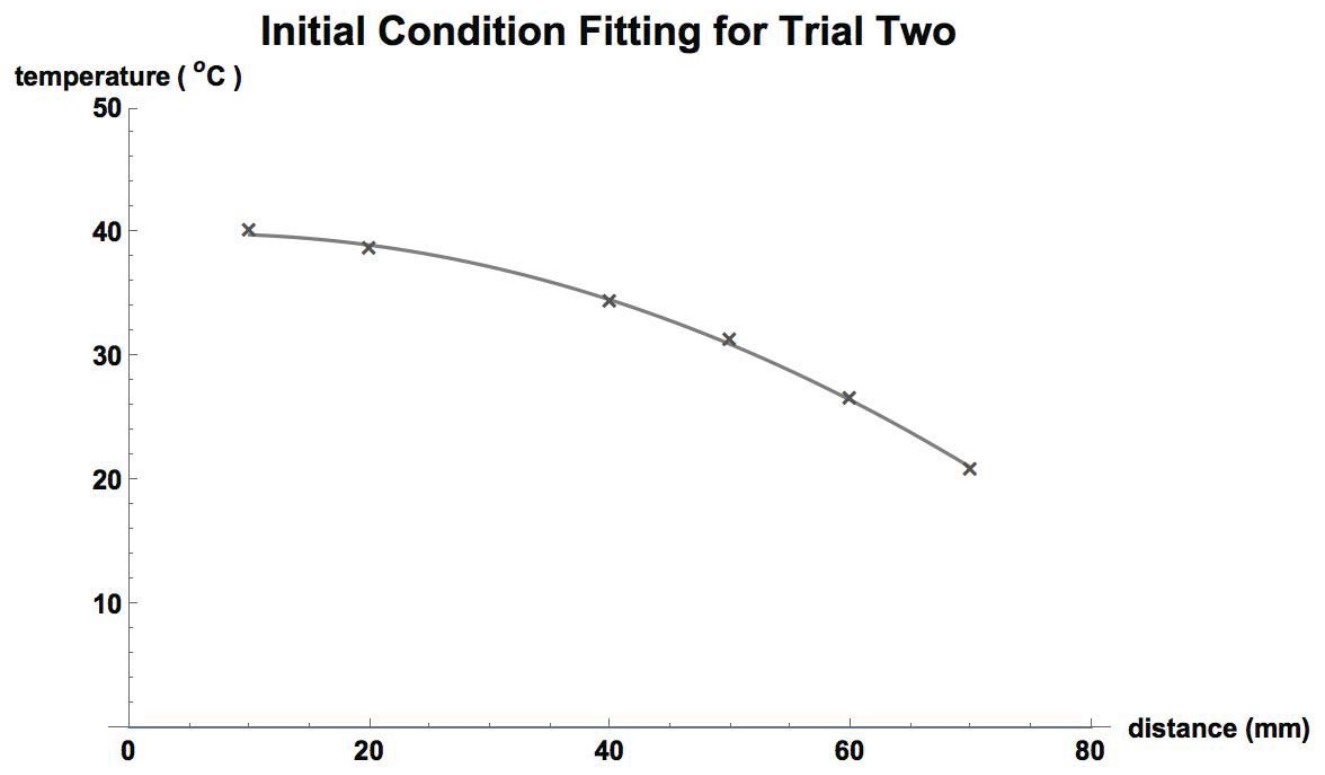

Figure 7. Trial 2 initial condition fitting from initial probe measurements across all thermistors as a function of radial distance from the center.

After finding fitting functions for the boundary and initial conditions, we have assembled all of the necessary information to construct our model:

$$
\begin{aligned}
& R_{i}=10 \mathrm{~mm} \\
& R_{o}=70 \mathrm{~mm} \\
& \kappa=37.52 \times 10^{-6} \frac{\mathrm{m}^{2}}{\mathrm{~s}} \\
& g_{1}(t)=-0.0461 t^{6}+0.6391 t^{5}-3.4099 t^{4}+8.4423 t^{3}-7.6973 t^{2}-6.1995 t+41.889 \\
& g_{2}(t)=-0.0314 t^{6}+0.4346 t^{5}-2.1891 t^{4}+4.1342 t^{3}+2.8109 t^{2}-24.435 t+59.484 \\
& h_{1}(t)=0.1897 t^{6}-2.7714 t^{5}+15.945 t^{4}-45.935 t^{3}+69.986 t^{2}-55.093 t+32.671 \\
& h_{2}(t)=0.5125 t^{6}-8.5651 t^{5}+57.822 t^{4}-201.41 t^{3}+381.78 t^{2}-375.47 t+165.64 \\
& v_{1}(r)=-0.0046(1000 r)^{2}+0.0551(1000 r)+39.776 \\
& v_{2}(r)=-0.0045(1000 r)^{2}+0.0541(1000 r)+39.698
\end{aligned}
$$

Before making use of the calculated condition functions, we must first form the basis functions that satisfy the heat equation in the cylindrical system. Since there is not an explicit formula for the eigenvalues of the equation, a finite list of basis functions must be created numerically. This is accomplished by calculating a set $\left\{m_{n}\right\}$ which are the square roots of the eigenvalues, a set of basis weights $\left\{B_{n}\right\}$, and a set of constants from the orthogonality principle $\left\{L_{n}\right\}$. Calculating the first thirty terms in these sets will lead us to generate the first thirty basis functions for the model. (Table 2) shows the calculations of these terms for $n=1,2,3,4,5$. 


\begin{tabular}{|c|c|c|c|}
\hline $\mathbf{n}$ & $\mathbf{m}_{\mathbf{n}}$ & $\mathbf{B}_{\mathbf{n}}$ & $\mathbf{L}_{\mathbf{n}} \times \mathbf{1 0}^{\mathbf{4}}$ \\
\hline 1 & 50.3 & 2.13 & 20.64 \\
\hline 2 & 103.4 & -6.57 & 80.85 \\
\hline 3 & 156.1 & -1.18 & 2.90 \\
\hline 4 & 208.7 & -0.34 & 1.02 \\
\hline 5 & 261.2 & 0.21 & 0.76 \\
\hline
\end{tabular}

Table 2. Table of square-root eigenvalues and weighting coefficients.

The values from (Table 2) allow the basis functions $Q(r)$ to be explicitly calculated. (Figure 8 ) verifies that these basis functions do indeed meet the necessary boundary conditions:

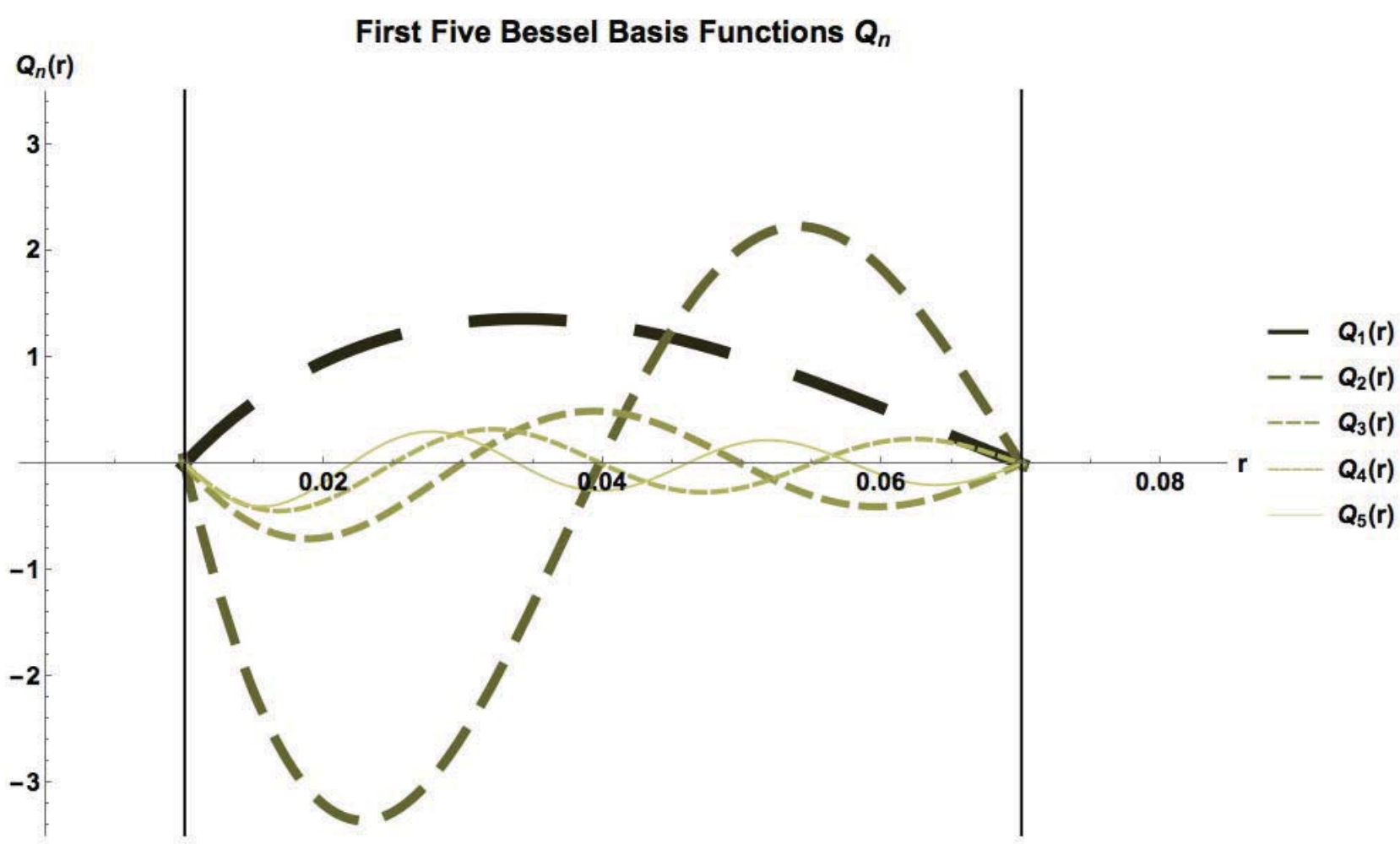

Figure 8. The first five $Q_{n}$ terms using the $m_{n}$ and $B_{n}$ values.

Vertical lines in the plot identify the inner and outer radii $R_{i}=10 \mathrm{~mm}$ and $R_{o}=70 \mathrm{~mm}$ respectively, and the colored curves represent the first five radial basis functions $Q_{n}$ based on the $m_{n}$ and $B_{n}$ values. Evidently the $m_{n}$ and $B_{n}$ values successfully produce a family of solutions satisfying both the radial ODE and the boundary conditions. This family of basis functions is then used to decompose $\hat{V}(r)$ and $\hat{F}(r, t)$.

All of the necessary information is present to successfully calculate the sets of coefficients in the solution $v(r, t)$ :

$$
\begin{gathered}
A_{n}=\int_{R_{i}}^{R_{o}} r \hat{V}(r) Q_{n}(r) d r \\
A_{n}(t)=\int_{0}^{t} \int_{R_{i}}^{R_{o}} r \hat{F}(r, \tau) Q_{n}(r) d r e^{m_{n}^{2} \kappa \tau} d \tau
\end{gathered}
$$


both of which substitute directly into the final form:

$$
v(r, t)=\sum_{n=1}^{30}\left[\left[A_{n}(t)+A_{n}\right]\left[J_{0}\left(m_{n} r\right)+B_{n} Y_{0}\left(m_{n} r\right)\right] e^{-m_{n}^{2} \kappa t}\right]+\frac{\ln (r)-\ln \left(R_{o}\right)}{\ln \left(R_{i}\right)-\ln \left(R_{o}\right)} g(t)+\frac{\ln \left(R_{i}\right)-\ln (r)}{\ln \left(R_{i}\right)-\ln \left(R_{o}\right)} h(t)
$$

The following figures verify the success of the Fourier-Bessel decomposition method for determining coefficients. (Figure 9) and (Figure 10) compare the initial condition functions for each trial with their respective thirty-term Fourier-Bessel expansions.

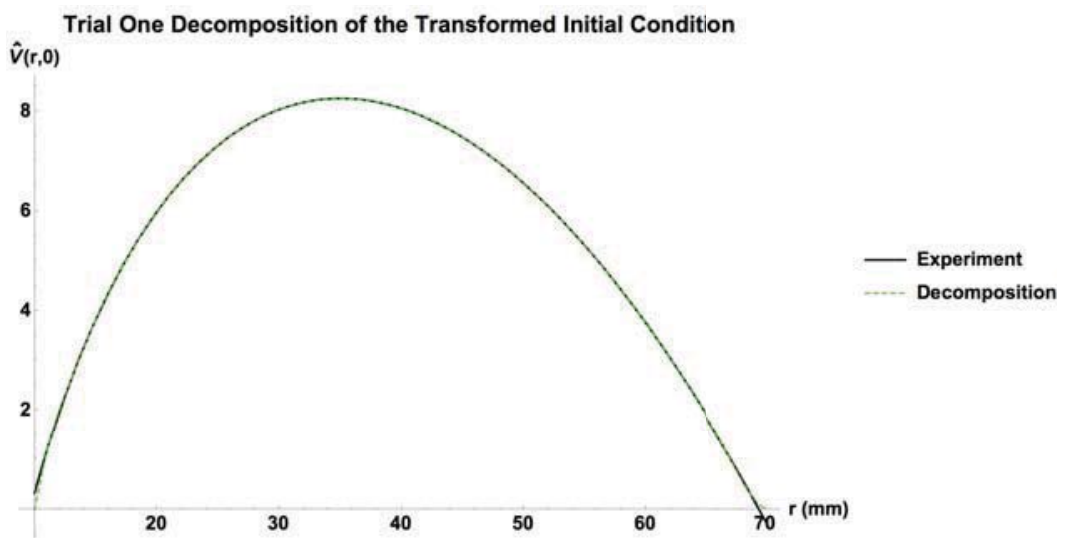

Figure 9. The 30 term decomposition of the transformed initial condition $\hat{V}(r)$ for trial 1.

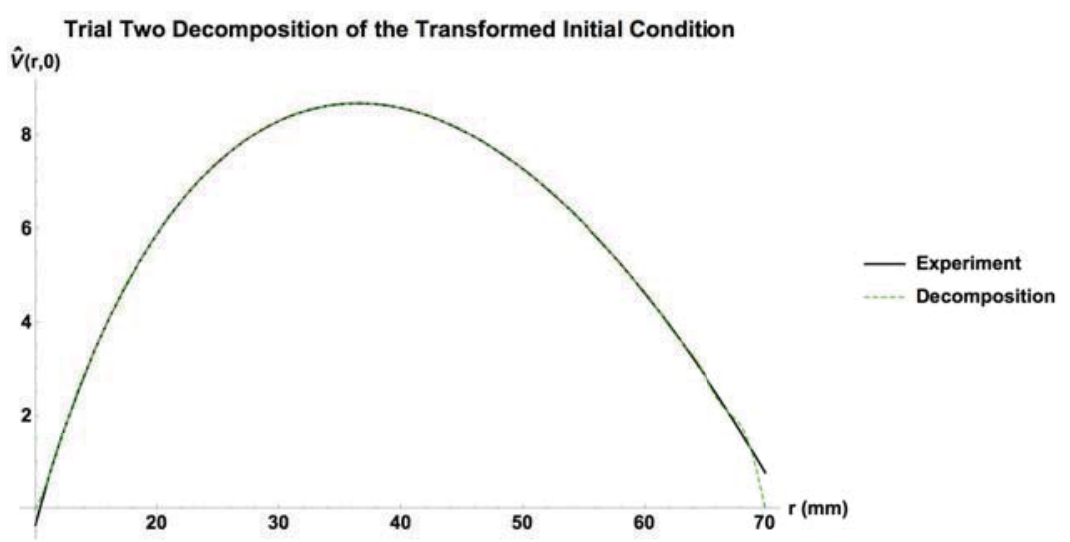

Figure 10. The 30 term decomposition of the transformed initial condition $\hat{V}(r)$ for trial 2.

This high degree of correlation between the initial conditions and their expansions inspires confidence in the decomposition method utilized in developing this model. The success of this explicit solution is shown graphically in (Figure 11) . Evidently, the $A_{n}$ coefficients determined from the Bessel-Fourier decomposition were accurate as the modeled heat solution matches closely with the intermediate thermistor measurements. With fewer than 30 terms of the sequence, we found that the model deviated further from the experimental data. As more terms are added to the model, the better the explicit solution agrees with the data.

Finally, the resulting analytical solution evaluated at the thermistor positions correlates to the measured data as shown in (Figure 11) and (Figure 12). Note that $T_{1}(r, t)$ and $T_{2}(r, t)$ replace $v(r, t)$ in the plots below to avoid ambiguity with the initial condition function $v(r)$ in (Figure 11), (Figure 12), (Figure 13), and (Figure 14). 


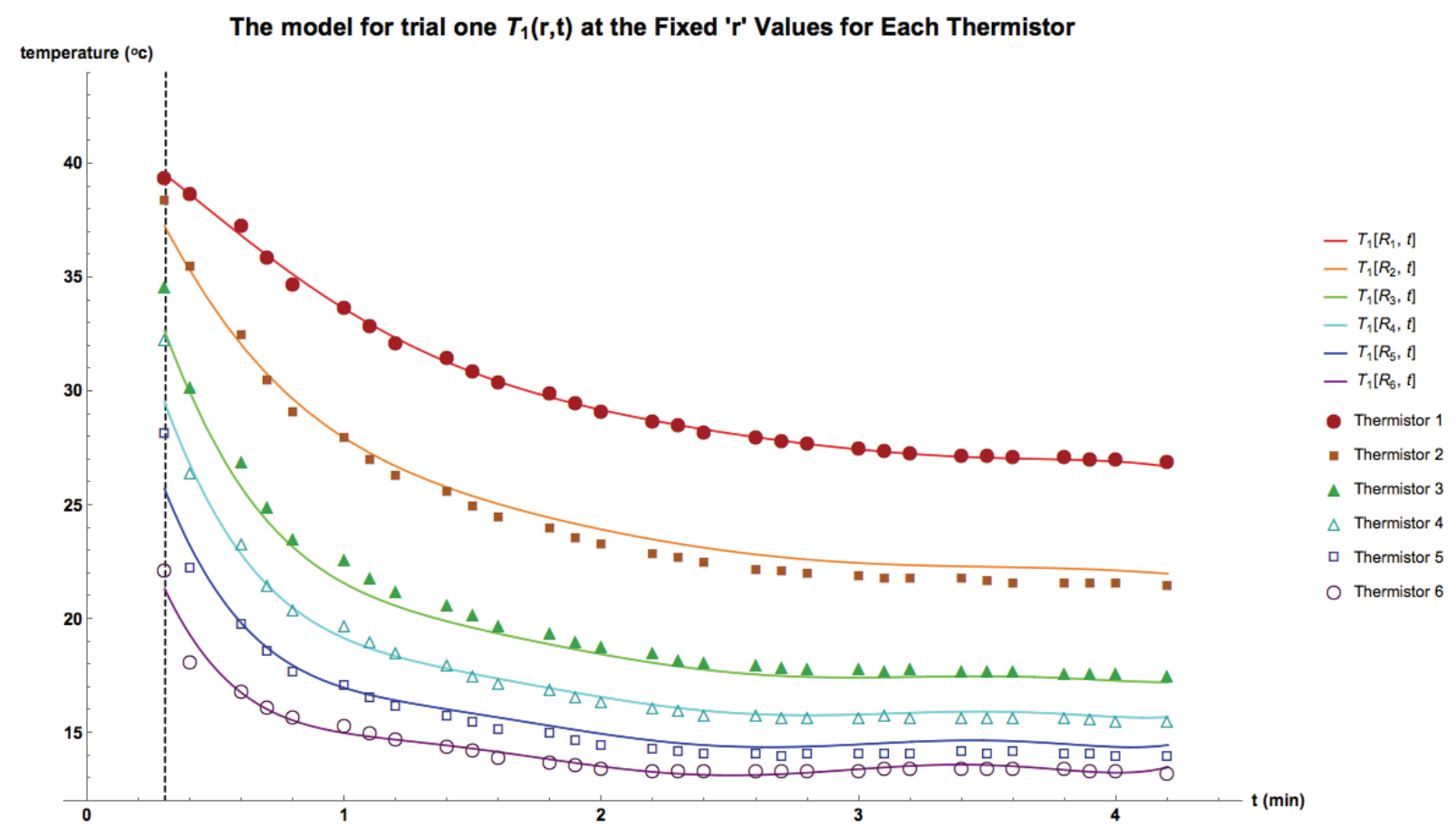

Figure 11. Trial 1 temperature data with analytic solution.

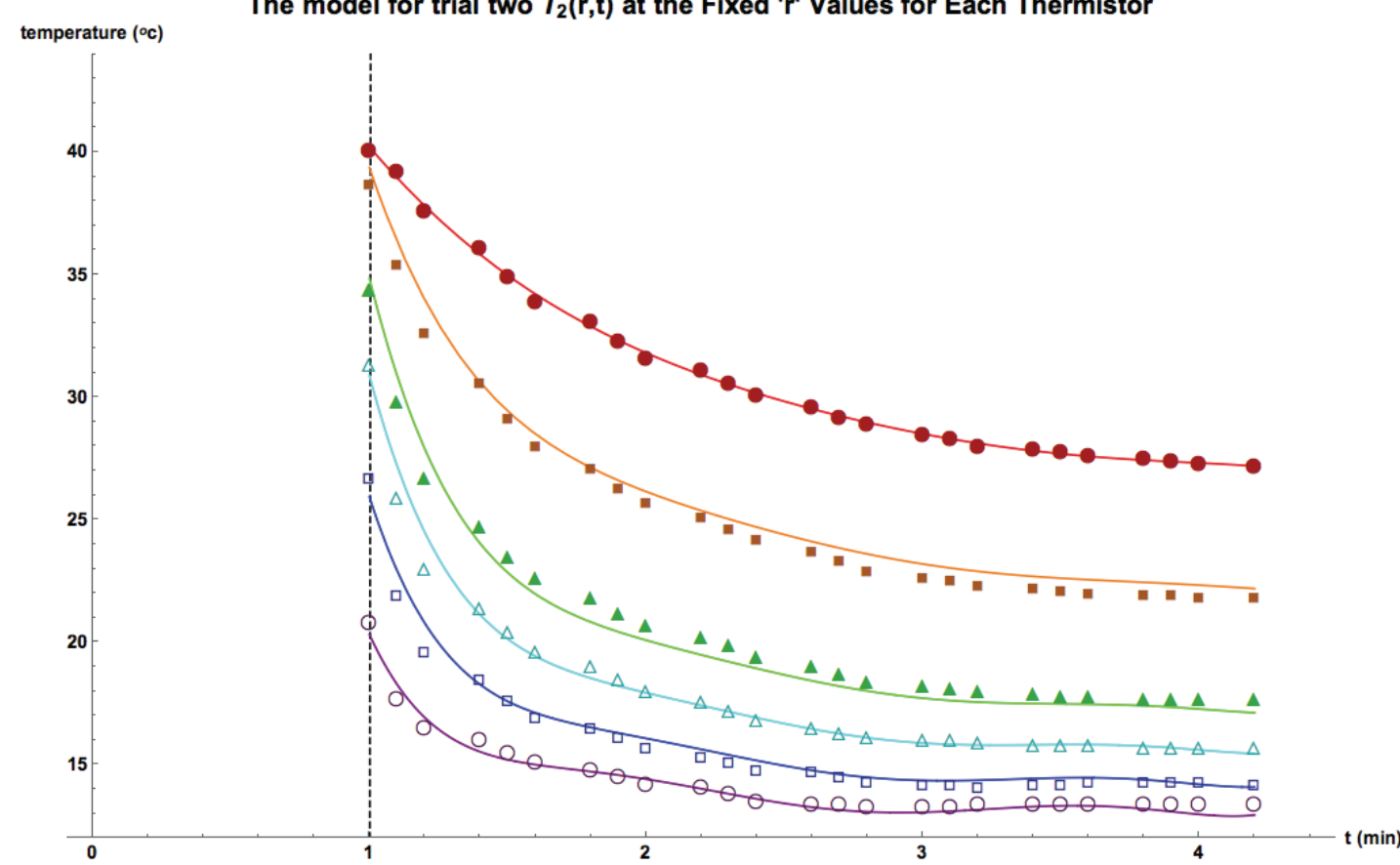

Figure 12. Trial 2 temperature data with analytic solution. 
Clearly, the mathematical models accurately describe the heat diffusion in the brass cylinder. For both trials, each color plot shows the time evolution of the temperature at the specific thermistors located a given distance from the center. As expected from the transform identity, the inner and outer radii solutions match up perfectly with the data. This can be seen by evaluating the solution at the boundary points $R_{i}, R_{o}$ :

$$
\begin{aligned}
& v\left(R_{i}, t\right)=\sum_{n=1}^{30}\left[\left[A_{n}(t)+A_{n}\right] Q_{n}\left(R_{i}\right) e^{-m_{n}^{2} \kappa t}\right]+\frac{\ln \left(R_{i}\right)-\ln \left(R_{o}\right)}{\ln \left(R_{i}\right)-\ln \left(R_{o}\right)} g(t)+\frac{\ln \left(R_{i}\right)-\ln \left(R_{i}\right)}{\ln \left(R_{i}\right)-\ln \left(R_{o}\right)} h(t)=g(t) \\
& v\left(R_{o}, t\right)=\sum_{n=1}^{30}\left[\left[A_{n}(t)+A_{n}\right] Q_{n}\left(R_{o}\right) e^{-m_{n}^{2} \kappa t}\right]+\frac{\ln \left(R_{i}\right)-\ln \left(R_{o}\right)}{\ln \left(R_{o}\right)-\ln \left(R_{o}\right)} g(t)+\frac{\ln \left(R_{i}\right)-\ln \left(R_{o}\right)}{\ln \left(R_{i}\right)-\ln \left(R_{o}\right)} h(t)=h(t)
\end{aligned}
$$

in which the strike-throughs indicate elements equal to zero. The remaining data set supports the success of our model.

\section{ANALYSIS}

To test the developed model, we tried varying the thermal diffusivity constant $\kappa$. This test was conducted by altering the thermal diffusivity constant from $\kappa=37.52 \times 10^{-6} \frac{\mathrm{m}}{\mathrm{s}^{2}}$ to $\kappa=57.52 \times 10^{-6} \frac{\mathrm{m}}{\mathrm{s}^{2}}$. The most illuminating method for viewing the alteration this causes is by comparing the initial condition quadratic fits from the experiment with the model's reconstruction of the initial condition. (Figure 11) and (Figure 12) clearly indicate that the models' initial conditions fit the data well as can be seen on the dashed lines. By switching the thermal diffusivity constant, the models' initial conditions fail to align as well with the initial quadratic fit function.

In (Figure 13) the left plots shows the alignment of the initial condition generated from the mathematical model to the experimental initial condition function using the proper thermal diffusivity for brass $\kappa=37.52 \times 10^{-6} \frac{\mathrm{m}}{\mathrm{s}^{2}}$. The right plot shows the alignment of the two functions for the modified thermal diffusivity of $\kappa=57.52 \times 10^{-6} \frac{\mathrm{m}}{\mathrm{s}^{2}}$.

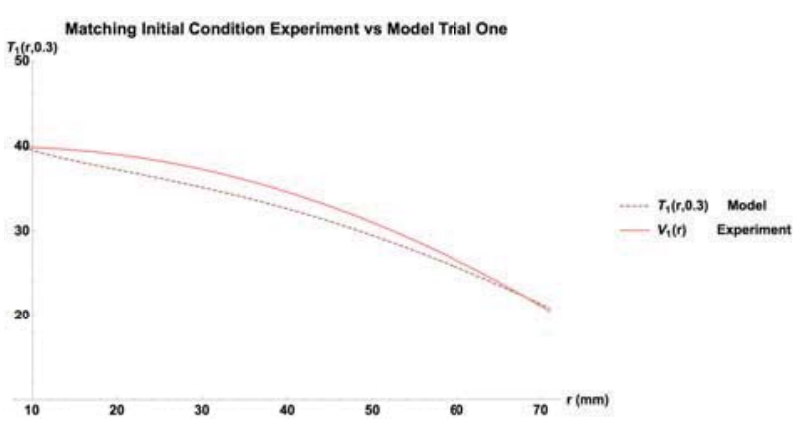

(a) $\kappa=37.52 \times 10^{-6} \frac{\mathrm{m}}{\mathrm{s}^{2}}$

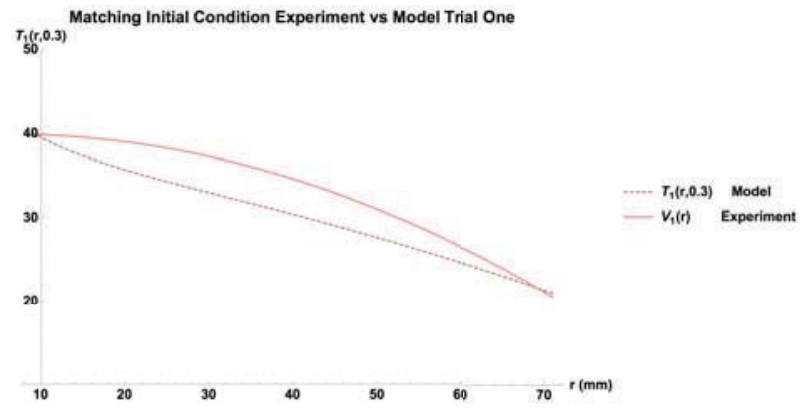

(b) $\kappa=57.52 \times 10^{-6} \frac{\mathrm{m}}{\mathrm{s}^{2}}$

Figure 13. Trial One Initial Condition Comparison with Varying Thermal Diffusivity $\kappa$

Similarly, (Figure 14) compares the alignment of initial conditions for the two different thermal diffusivity constants. 


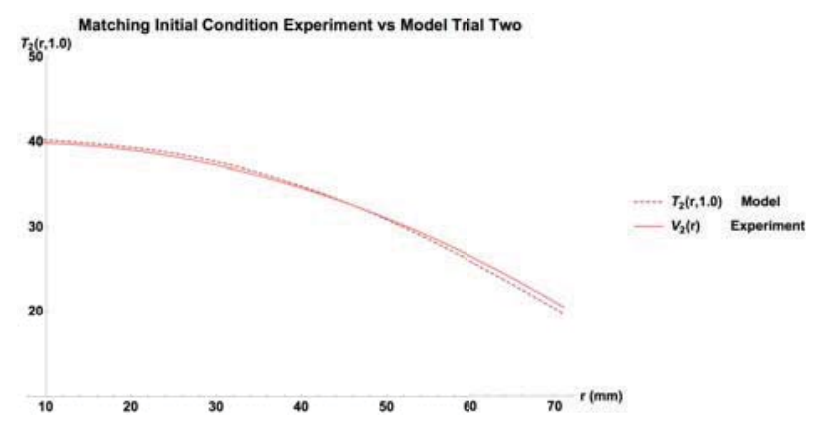

(a) $\kappa=37.52 \times 10^{-6} \frac{\mathrm{m}}{\mathrm{s}^{2}}$

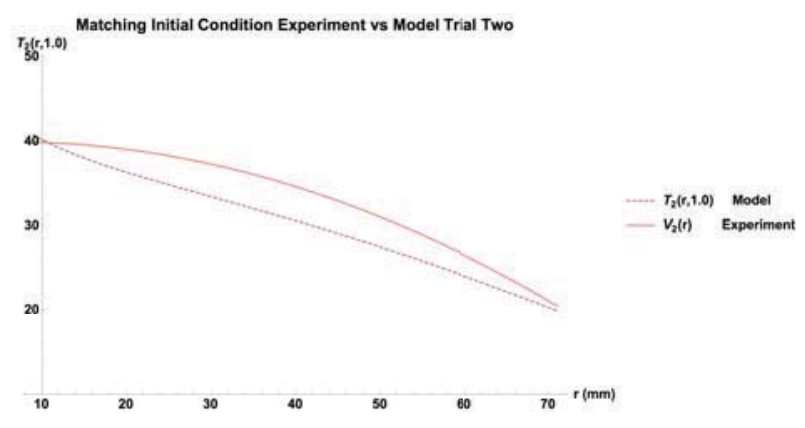

(b) $\kappa=57.52 \times 10^{-6} \frac{\mathrm{m}}{\mathrm{s}^{2}}$

Figure 14. Trial Two Initial Condition Comparison with Varying Thermal Diffusivity $\kappa$

Notice both (Figure 13) and (Figure 14) illustrate that altering the thermal diffusivity constant significantly reduces the success of the model to match the initial condition data. The altered diffusivity constant was increased by approximately $53 \%$ and this change substantially increased the discrepancy between the model and the experiment. Not only does our developed model accurately simulate the experiment, it also confirms the thermal diffusivity constant chosen for brass.

\section{CONCLUSION}

The results in (Figure 11) and (Figure 12) deeply support the agreement of the explicit method for solving the heat equation in cylindrical coordinates with time-varying boundary conditions with empirical data.

To construct the explicit solution it was necessary to approximate the boundary conditions of the cylinder using polynomial fits on the inner and outer thermistor data sets, which intrinsically each have their own error with respect to the data. The error metric we use to evaluate the success of our model is the relative error based on the square difference error at each point. Explicitly we use the formula

$$
E\left(r_{n}\right)=\frac{\sqrt{\sum_{t=0}^{t_{\text {final }}}\left(p\left(r_{n}, t\right)-v\left(r_{n}, t\right)\right)^{2}}}{\sqrt{\sum_{t=0}^{t_{\text {final }}} p\left(r_{n}, t\right)^{2}}}
$$

in which $r_{n}$ is the chosen thermistor ( $r_{1}$ is the innermost thermistor and thus indicative of the inner boundary condition) for the error metric, $t$ is an index of the times data were collected, $p\left(r_{n}, t\right)$ is the collected thermistor data, and $v\left(r_{n}, t\right)$ is the explicit solution. For trial one the errors were found for the inner and outer boundary conditions: $1.9 \%$ error on the inner boundary condition (red curve) and $8.8 \%$ error on the outer boundary condition (purple curve). So the errors on the explicit solution were $4.7 \%$ for $r_{2}, 6.3 \%$ for $r_{3}, 6.3 \%$ for $r_{4}$, and $7.9 \%$ for $r_{5}$. All of these errors were between the two errors accounted with the boundary condition fits, which suggests our explicit solution accurately fits with the thermistor data. The involved process of solving the heat diffusion problem by building a Green's function and then utilizing variation of parameters proves to be rigorous and accurate. This accuracy is further verified by the observed convergence to the measured data after only 30 terms in the Fourier-Bessel decomposition. In the future, other physical partial differential equations with time variant boundaries may be solved by applying this method of PDE transformation and solving the inhomogeneous equation by constructing a Green's function and then applying variation of parameters.

\section{ACKNOWLEDGEMENTS}

We thank Professor Harvey Segur for his devoted effort and insightful advice throughout the development of our project. We also thank the Department of Applied Mathematics at the University of Colorado, Boulder for generously providing the experimental resources to make this project possible. 


\section{REFERENCES}

1. Pinsky, M. (1991) Partial differential equations and boundary-value problems with applications 2nd ed., pp 171-235, McGraw-Hill, New York.

2. Pinsky, M. (1991) Partial differential equations and boundary-value problems with applications 2nd ed., pp 427- 465, McGraw-Hill, New York.

3. Laskar, J.M., Bagavathiappan, S., Sardar, M., Jayakumar, T., Philip, J., and Raj, B. (2008) Measurement of thermal diffusivity of solids using infrared thermography, Mater. Lett. 62, 2740-2742.

4. Pinsky, M. (1991) Fourier Series, in Partial Differential Equations and Boundary-Value Problems with Applications, (Sally, P., Su, F., Silverman, J., \& Tolman, S. Eds.) 2nd ed., 35-45, American Mathematical Society, Rhode Island.

5. Pinsky, M. (1991) Appendixes, in Partial Differential Equations and Boundary-Value Problems with Applications, (Sally, P., Su, F., Silverman, J., \& Tolman, S. Eds.) 2nd ed., 465-476, American Mathematical Society, Rhode Island.

6. Pinsky, M. (1991) Preliminaries, in Partial Differential Equations and Boundary-Value Problems with Applications, (Sally, P., Su, F., Silverman, J., \& Tolman, S. Eds.) 2nd ed., 10-20, American Mathematical Society, Rhode Island.

7. Pinsky, M. (1991) Boundary-Value Problems in Cylindrical Coordinates, in Partial Differential Equations and BoundaryValue Problems with Applications, (Sally, P., Su, F., Silverman, J., \& Tolman, S. Eds.) 2nd ed., pp 183, American Mathematical Society, Rhode Island.

8. Abramowitz, M., \& Stegun, I. A. (1964). Handbook of mathematical functions with formulas, graphs, and mathematical tables, pp 374 - 376 , U.S. Govt. Print. Off., Washington, DC.

9. Abramowitz, M., \& Stegun, I. A. (1964). Handbook of mathematical functions with formulas, graphs, and mathematical tables, pp 358 - 363, U.S. Govt. Print. Off., Washington, DC.

10. Pinsky, M. (1991) Appendixes, in Partial Differential Equations and Boundary-Value Problems with Applications, (Sally, P., Su, F., Silverman, J., \& Tolman, S. Eds.) 2nd ed., pp 465-468, American Mathematical Society, Rhode Island.

\section{ABOUT THE STUDENT AUTHORS}

Kaitlyn Parsons graduated from the University of Colorado, Boulder with two Bachelor of Science degrees in Engineering Physics and Applied Mathematics in May 2016. Kaitlyn will obtain her PhD in materials science and engineering.

Tyler Reichanadter is at the University of Colorado, Boulder working towards a double major in Applied Mathematics and Engineering Physics. After finishing both of his BS degrees, Tyler intends to obtain his Masters in Applied Mathematics at CU Boulder.

Andi Vicksman is starting her senior year as a General Engineering and Applied Mathematics double major at University of Colorado Boulder. In General Engineering coursework her emphasis is on Civil Engineering, while also getting a Secondary Math Teaching License. She has a passion for education and hopes to teach engineering in K-12 after graduation.

\section{PRESS SUMMARY}

The heat equation is a partial differential equation that elegantly describes heat conduction or other diffusive processes. Primary methods for solving the heat equation are only applicable when the boundary conditions do not change with time. In this paper, we analyzed a physical system in which a solid, brass cylinder experiences heat flow from the central axis to a heat sink along its outer rim. We constructed an explicit mathematical framework by which to handle the heat equation with these time-variant boundary conditions. Experimental results verify the success of this analytical method. 